\title{
Dépenses publiques en capital humain et croissance économique au Mali
}

\author{
Amadou BAMBA ${ }^{1}$, Yaya SIDIBE ${ }^{2}$, Sory DOLO ${ }^{3}$ et Mamady SISSOKO ${ }^{4}$ \\ ${ }^{1}$ Université des Sciences Sociales et de Gestion de Bamako (USSGB), Auteur de correspondance \\ ${ }^{2}$ Université des Sciences Sociales et de Gestion de Bamako (USSGB) \\ ${ }^{3}$ Université des Sciences Sociales et de Gestion de Bamako (USSGB) \\ ${ }^{4}$ Université des Sciences Sociales et de Gestion de Bamako (USSGB), Pharmacie Populaire du \\ Mali
}

\begin{abstract}
Résumé : L'objectif de ce papier est de mesurer l'effet des dépenses publiques en capital humain sur la croissance économique au Mali. Les données couvrent la période de 1986 à 2018. A l'aide de la méthode d'estimation ARDL, les résultats montrent que les dépenses en capital humain sont favorables à la croissance économique de l'année en cours et moins favorables pour les années qui suivent. Dans le long terme, les dépenses en capital humain ont un effet positif sur la croissance économique au Mali, l'effet est plus important pour les dépenses de santé que les dépenses d'éducation. Au regard de ces résultats, les politiques économiques doivent porter sur, l'orientation des dépenses en capital humain vers les dépenses d'investissements dans le secteur de la santé et de l'éducation.
\end{abstract}

Mots clés : Dépenses publiques - Capital humain - Croissance économique - Mali.

Abstract: The objective of this paper is to measure the effect of public spending on human capital on economic growth in Mali. The data cover the period from 1986 to 2018. Using the ARDL estimation method, the results show that human capital expenditure is favorable to economic growth in the current year and less favorable for the years that follow. In the long run, spending on human capital has a positive effect on economic growth in Mali, the effect being larger for health spending than education spending. In view of these results, economic policies should focus on the orientation of human capital expenditure towards investment expenditure in the health and education sector.

Keywords: Public expenditure - Human capital - Economic growth - Mali 


\section{Introduction}

L'analyse du rôle productif des dépenses a connu un développement dans les années 40-50, à la suite des débats autour de la croissance équilibrée initiés par les théoriciens du développement (Rosenstein-Ridan, 1943; Hirschman et Sirkin, 1958). Ces débats ont néanmoins été rapidement éclipsés à partir des années 60, la réflexion se déplaçant sur le front des questions de court terme et de l'opposition entre effet multiplicateur et effet d'éviction des dépenses publiques. Pendant plus de trente ans, les dépenses publiques sont perçues comme un facteur de relance de la demande dans une optique de tradition Keynésienne, et son rôle productif occulté. La perception du rôle des dépenses publiques comme facteur de croissance économique a remarquablement évolué au cours de ces dernières années. On assiste actuellement à un renouveau de l'analyse des effets de ces dépenses sur la croissance économique, en particulier grâce aux théories de la croissance endogène qui mettent l'accent sur les externalités positives qu'engendrent certains aménagements publics.

En effet, le modèle de Solow (1956) concluait que la croissance économique à long terme est expliquée par les facteurs capital, travail et le progrès technique. La recherche des facteurs expliquant le progrès technique aboutit à l'intégration du capital humain comme un facteur déterminant de la croissance économique par les théoriciens de la croissance économique. Les auteurs tels Mankiw, Romer et Weil (1992), Benhabib et Spiegel (1994) et bien d'autres considèrent le capital humain comme variable principale de l'évolution macroéconomique. Les théoriciens du capital humain, Schultz (1961) et Becker (1964), tous prix Nobel placent le capital humain au cœur de l'amélioration de la productivité ; ils attestent qu'un niveau plus élevé d'éducation améliore la productivité des travailleurs. C'est pourquoi les pays investissent plus dans l'éducation et la santé, deux éléments clés du capital humain. Ces dépenses sont des sources de développement et de l'amélioration de la qualité du capital humain, ce qui permet de contribuer positivement au élancement de la croissance économique. Les gouvernements des pays en développement, comme le Mali, ont souvent accordé une faible part des dépenses publiques à l'amélioration de la santé publique et de l'éducation. Dans pratiquement tous les pays en développement, le ministère de la santé et de l'éducation ont très souvent, dans l'affectation des crédits budgétaires des parts assez faibles. Toutefois, la tendance s'est partiellement inversée au cours des vingt-cinq dernières années. Dans les années 70 , la santé et le développement ont suscité un intérêt accru, du fait de l'attention portée aux stratégies de développement fondées sur l'équité, puis en raison des inquiétudes provoquées par les effets du ralentissement de la croissance économique, dans les années 80 . Toute la période a été marqué par un renforcement de l'opinion selon les dépenses de santé constituaient, comme celles de l'éducation, des investissements dans le capital humain.

Les dirigeants africains ont accepté en 2001 par la Déclaration d'Abuja d'allouer 15\% de leur budget national aux dépenses de santé, mais en 2013, seuls cinq (5) pays africains ont atteint cet objectif (Banque mondiale, 2014). Au Mali, en dépit de la situation difficile en matière de sécurité, l'économie malienne a obtenu des résultats raisonnables au cours des dernières années. La croissance réelle a avoisiné en moyenne $5 \%$ par an et l'inflation a été bien maîtrisée grâce à l'arrimage du franc CFA à l'euro (Rapport PEFA, 2019). Selon le FMI (2019), la croissance du PIB en termes réels a été estimée à 5,8\% en 2016, à 5,4\% en 2017 et 4,7 en 2018. Quant au taux d'inflation, il a été de $2,1 \%$ en 2018 contre $1,9 \%$ en 2017. Cependant, comme la population progresse d'environ $3 \%$ par an, la croissance annuelle du PIB par habitant est modeste. L'analyse de la politique des dépenses sous l'angle de la classification fonctionnelle fait ressortir une progression de la fonction Enseignement liée à l'accroissement de l'effectif des 
élèves et des recrutements d'enseignants en vue d'atteindre les Objectifs du Millénaire pour le Développement (OMD) devenu aujourd'hui les Objectifs de Développement Durable (ODD); cela fait augmenter les dépenses en éducation. Aussi, la fonction Santé connaît une hausse imputable aux dépenses liées au renforcement d'effectifs du personnel sanitaire, aux subventions accordées aux formations sanitaires dans le cadre de leur mission de service public, à la lutte contre les maladies et à la prise en charge des politiques de gratuité des soins de certaines pathologies (Rapport PEFA, 2019).

Le Gouvernement du Mali considère l'éducation et la santé comme le troisième pilier de sa stratégie de croissance accélérée et de réduction de la pauvreté. L'accroissement des allocations budgétaires au niveau de ces deux secteurs ne se traduit par toujours leur meilleure performance. En effet, les ressources allouées peuvent ne pas parvenir - ou arriver très en retard - au destinataire final à cause de l'existence de goulots d'étranglement dans le système d'acheminement des ressources. Cependant, les investissements dans ces secteurs permettent, selon les théoriciens du capital humain d'augmenter la productivité des personnes et donc la croissance économique (Becker, 1964). C'est sans doute ce qui motive le gouvernement malien à utiliser ces deux secteurs comme pilier pour réduire la pauvreté. En 39 ans les dépenses d'éducation en pourcentage du PIB ont augmenté de 6\% en moyenne. Sur la période 19782017, on enregistre une moyenne annuelle de 3,32. Le changement enregistré entre cette période est de $6 \%$. C'est en 2017 qu'on enregistre la valeur la plus élevée $(3,787)$ et c'est en 1978 qu'on enregistre la valeur la plus basse $(3,56)$ (Banque Mondiale, 2021). La part de la santé dans le budget de l'Etat augmente depuis quelques années, pour s'établir actuellement à 8,4\% des dotations totales. La principale source de financement du PRODESS (Programme de Développement Socio-sanitaire) vient du budget de l'Etat. Au Mali, la couverture en infrastructures et en personnel santé s'est améliorée depuis le début de la mise en œuvre du PRODESS en particulier dans les régions initialement déshéritées. Les progrès au niveau des piliers du système de santé ont permis d'améliorer l'accessibilité des populations à $5 \mathrm{~km}$ de la population à un centre de santé fonctionnel est passée de 44\% en 2002 à $57 \%$ en 2009. Il en est de même pour les dépenses d'éducation qui ont aussi accrus avec les politiques d'éducation pour tous débutées en 2000 avec le PRODEC (Programme Décennal de Développement de l'Education).

Malgré tous ces efforts d'investissement en capital humain, la croissance économique du Mali tourne en moyenne autour de 5\%. L'originalité de notre article est qu'il permet de savoir, après tant d'efforts fournis pour augmenter les dépenses de santé et d'éducation à travers les programmes décennaux par le Mali, de connaitre approximativement leurs effets sur la croissance économique. Cela va éclairer les décideurs de politiques économiques sur l'effet des dépenses en capital humain sur la croissance économique du Mali. Le reste de l'article portera sur l'état de la revue de littérature, la méthodologie, les résultats suivie de la conclusion et la bibliographie.

\section{Revue de littérature}

Cette revue de la littérature est subdivisée en revue théorique et empirique.

\subsection{Littérature théorique}

La question fondamentale en économie de développement est, pourquoi certains pays sont pauvres et d'autres sont riches ? Smith (1776) avait déjà souligné dans la richesse des nations l'importance du capital humain et que l'investissement dans le capital humain permettait d'accroitre la 
productivité future. Cela a été approfondi plus tard par les théoriciens du capital humain, notamment (Mincer, 1974 ; Becker, 1975), les personnes les plus instruites et en bonne santé ont une plus grande chance de participer au marché du travail ; ce qui réduit, si elles sont économiquement actives, leurs probabilités d'être au chômage. Dans les premiers modèles de croissance développés par Solow (1956), la croissance de long terme dépendait du progrès technique sans que son origine soit réellement analysée. C'est à la recherche de ce progrès à travers les facteurs de la croissance économique que les travaux de Romer (1990) et de Lucas (1988) se sont focalisés. Ces auteurs ont démontré, sur des bases analytiques plus rigoureuses, que les pays, dont les populations ont davantage bénéficié d'un plus grand effort éducatif, sont devenus plus riches. Même si le rôle de l'éducation est établi, les mécanismes par lesquels elle agit sur la croissance économique restent à identifier. Les investissements dans l'éducation et la santé des pauvres ont des effets bénéfiques sur la productivité, le revenu et sur la croissance économique (Diagne, 2007). C'est ce qui explique selon lui ; l'accent mis sur ces deux secteurs dans les stratégies de lutte contre la pauvreté. La prise en compte du capital humain comme facteurs de croissance n'est pas spécifique aux modèles de croissance. Les travaux d'analyse de Maddison, 1987 menés dans la tradition de Solow, intègrent un indice de « qualité de la main d'œuvre» qui multiplie l'efficacité de celle-ci. Le modèle de Lucas (1988) stipule sur l'hypothèse habituelle de la théorie du capital humain selon laquelle le capital humain de l'ensemble des agents peut être agencé en un stock unique, comme l'est par ailleurs le capital physique. De ce fait, le modèle de Lucas situe la source de la croissance dans l'accumulation de capital humain effectué par des individus dans le cadre de rendements croissants. Si le modèle de Lucas introduit directement le capital humain comme facteur de production de biens finals, le modèle de Romer donne au capital humain un rôle un peu diffèrent. Selon l'auteur, l'innovation technologique est source de croissance et procure en plus un pouvoir de monopole qui est l'incitation première à mettre en œuvre les nouveaux procédés.

Pour le modèle d'Aghion et Howitt (1992), qui soit une réduction des coûts de production, soit un accroissement de la qualité des produits, entraîne une hausse de la productivité pour toute l'économie et pour l'avenir. Grossman (1999) applique le concept de «capital humain », de cycle de vie et d'investissement à la demande de santé et de soins, qui selon lui, correspondrait à un investissement de la part des individus, soucieux d'augmenter à la fois leur qualité de vie et leur capacité de travail et partant leur revenu. Behrman (1998) a analysé comment la croissance et la santé interagissent sur le plan théorique ; la santé est une composante essentielle du capital humain, les investissements dans la santé ont des effets directs et indirects sur la productivité et par voie des conséquences sur la croissance économique. Dans la majorité des pays en développement, les investissements publics représentent une part élevée des dépenses totales, en raison du rôle joué par les pouvoirs publics dans l'offre d'infrastructures, par exemple dans des domaines des transports, santé, des télécommunications et de l'énergie ; plusieurs travaux de recherche ont visé à évaluer la contribution des dépenses publiques de transport à la croissance. L'amélioration de la santé contribue à la croissance économique et de plusieurs manières : elle limite le manque à produire imputable à l'incidence de la morbidité sur la main d'œuvre, elle permet d'exploiter des ressources naturelles qui, situées dans des zones infestées de vecteurs d'agents pathogènes, étaient totalement ou largement inaccessibles ; enfin, elle libère à d'autres fins les ressources qui auraient servi, sinon à soigner les malades. Les effets les plus évidents de l'amélioration de la santé sur la population sont la réduction du nombre de journées perdues pour cause de maladie, l'augmentation de la productivité, l'amélioration des chances d'accéder à des emplois mieux rémunérés et l'allongement de la durée de vie active. 
Dans les années 1980 et 1990, l'éducation a été mis en exergue dans la théorie de la croissance endogène considérant l'éducation comme un processus qui modifie la technologie de production elle-même (Romer, 1990 ; Aghion et Howitt, 1999), facilite l'adaptation des technologies etrangères (Barro, 1999 ; Sala-i-Martin, 1997 ; Hall et Jones, 1999). Elle facilite aussi le transfert de ressources vers le secteur le plus technologiquement dynamique de l'économie (Schiff et Wang, 2004). Il apparait que dans le modèle de croissance néoclassique élargi (Mankiw, Romer et Weil, 1992), l'éducation doit avoir un effet positif sur le taux de croissance du revenu. Cependant, il est possible qu'un niveau minimum d'éducation soit requis pour que l'éducation ait un impact mesurable sur la croissance (Rebelo, 1991). Selon Grossman (1972), la santé est considérée comme un bien d'équipement, puisque la production de santé détermine le temps consacré au travail. Les personnes en meilleure santé sont moins susceptibles de s'absenter au travail pour cause de maladie et sont donc plus productives. Les tenants de la nouvelle théorie de la croissance économique, auxquels sont associés les auteurs (Romer, 1986 ; Barro, 1991et Lucas, 1988) ont ravivé l'intérêt de l'étude du capital comme un facteur déterminant de la croissance économique

Le Programme des Nations Unies pour le Développement (PNUD, 1994) indique que le développement des infrastructures hospitalières et le niveau de l'éducation de la population, a permis de reculer la mortalité entraînant une augmentation de l'espérance de vie de la population. Le PNUD insiste sur le fait que l'investissement dans la construction des centres de santé universitaires contribue à développer la recherche afin de soigner d'avantage la population ; en fait, dans les pays en développement, les dépenses allouées aux services de santé ne sont pas accessibles à une large partie de la population. De ce fait, la construction des centres de santé devient un indicateur important dans la relation entre la santé et la croissance économique. Mais cet indicateur peut être biaisé dans la mesure où les centres hospitaliers universitaires sont le plus souvent construits dans les universités, on pourrait prendre plutôt en compte la construction des centres de santé et hôpitaux dans les zones reculées. Dans la majorité des pays en développement, les investissements publics représentent une part élevée des dépenses totales, en raison du rôle joué par les pouvoirs publics dans l'offre d'infrastructures, par exemple dans des domaines d'éducation, de transport, des télécommunications et de l'énergie; plusieurs travaux de recherche ont visé à évaluer la contribution des dépenses publiques de santé à la croissance.

\subsection{Littérature empirique}

La recherche, le capital humain et les dépenses publiques sont les principaux facteurs qui expliquent la croissance endogène (Diagne, 2007). Or, selon l'auteur, la santé et l'éducation sont les seuls secteurs qui intègrent ces facteurs. Le capital humain apparait donc comme un facteur clé de la croissance économique. La relation entre la croissance économique et le capital humain est le plus souvent traitée dans la littérature par des régressions pour les différents pays avec plusieurs autres variables portant sur le capital physique, l'environnement. Denison (1961) attribue $23 \%$ de la croissance économique des USA, de 1930 à 1960, à l'amélioration du secteur éducatif. Les premiers travaux empiriques mettant en exergue la relation entre le secteur productif et le secteur éducatif sont attribués à Romer (1986) et Lucas (1988). Mais, c'est sans aucun doute Schultz qui a fait observer pour la première fois, dans un article publié en 1961, que le facteur travail a toujours été pris en compte par les économistes pour expliquer l'origine de la richesse des nations. Schultz (1961) de l'école de Chicago a écrit : « Considérez à la fois les compétences innées et acquises. Celles-ci sont importantes et peuvent être investies pour se développer, elles formeront le capital humain ». L'auteur élargit la signification de 
l'investissement pour inclure toutes les activités qui améliorent les compétences et la productivité d'un individu, notamment les dépenses de santé, la formation continue, l'éducation formelle, les programmes d'études pour adultes (comme les extensions agricoles) et la migration. C'est à la suite que l'auteur pionnier de la théorie du capital humain Becker (1964) développe tout une base théorique montrant l'importance de l'investissement dans le capital humain. Pour Becker, le capital humain est « tout investissement dans l'éducation, la formation, les compétences, la santé et d'autres valeurs qui ne peuvent être séparées de l'individu ». La littérature expliquant la relation capital humain et croissance va se développer différemment en fonction de la mesure du capital humain et des mécanismes pour ce dernier à avoir un effet sur la croissance économique.

Issolah et al., (2021) montrent que le développement humain est un instrument de mesure et d'évaluation du degré de succès des pays à satisfaire les besoins socio-économiques des populations. Ils analysent l'impact du développement du capital humain sur la croissance économique à l'aide de modèle ARDL en Algérie de 1986 à 2017. Ils parviennent à la conclusion qu'il existe une relation positive entre les taux de scolarisation et un effet négatif des dépenses de santé sur la croissance économique. Dans leur étude sur la contribution du capital humain sur la croissance économique au Maroc, Samoud et Driss (2021) utilisent un modèle à retard échelonné reliant le capital humain, l'investissement et la santé à la croissance économique de 1990 à 2019. Les résultats empiriques mettent en exergue le rôle déterminant et l'effet positif des différents aspects du capital humain sur la croissance économique au Maroc, et ce, dans un long et court terme. El Houda Sadi, et Rezine, (2021) trouvent à l'aide des données de panel sur l'Algerie, la Tunisie, l'Iran, la Jordanie et l'Egypte un impact positif et significatif de l'investissement en capital fixe, de l'enseignement primaire et secondaire sur le PIB réel. Bathily et Gueye (2021) étudient la relation entre dépenses publiques de santé et la croissance économique dans 25 pays d'Afrique Subsaharienne. A l'aide des panels VAR sur les données de 1996 à 2016, les auteurs trouvent que l'augmentation des dépenses publiques de santé peut entrainer une amélioration des résultats sanitaires à travers l'amélioration de l'espérance de vie et par ricochet la croissance économique dans le long terme.

Piabuo et Tieguhong (2017) analysent l'impact des dépenses de santé entre les pays de la sousrégion CEMAC et cinq autres pays africains. Ils utilisent les techniques d'estimation de panel des moindres carrés ordinaires (OLS) moindres carrés ordinaires entièrement modifiés (FMOLS) et les moindres carrés ordinaires dynamiques (DOLS). Les résultats ont montré que les dépenses de santé ont un effet positif et significatif sur la croissance économique dans les deux échantillons. Un changement d'unité dans les dépenses de santé peut potentiellement augmenter le PIB par habitant de 0,38 et 0,3 unité pour les cinq autres pays africains qui atteignent respectivement l'objectif d'Abuja et pour les pays de la CEMAC, une différence significative de 0,08 unité entre les deux échantillons. En outre, il existe également une relation à long terme entre les dépenses de santé et la croissance économique pour les deux groupes de pays. Mohapatra (2017) examine par une approche en deux étapes pour étudier le lien causal bidirectionnel entre la croissance économique et les dépenses publiques ; dépenses publiques de santé et le taux de mortalité infantile : et la croissance économique et le taux de mortalité infantile (IMR) dans le contexte indien. Les résultats suggèrent que le PIB de Granger entraîne les dépenses publiques de santé à court terme et à long terme, mais les dépenses publiques de santé pour Granger ne provoquent le PIB qu'à long terme. En outre, les dépenses publiques pour la santé et la croissance économique ont été trouvées à Granger à cause d'IMR à long terme. Cependant, le lien inverse ente le taux de mortalité infantile et les dépenses publiques de santé et/ou de croissance économique n'étaient pas significatives. Dinçer et Yuksel (2019) 
identifier la relation de causalité entre les dépenses de santé et la croissance économique dans les économies émergentes (Chine, Inde, Brésil, Russie, Mexique, Indonésie et Turquie). Ils utilisent la méthode de cointégration du panel de Pedroni et l'analyse de causalité du panel de Dumitrescu Hurlin pour les années entre 1996 et 2016. À la suite du test de cointégration du panel de Pedroni, les auteurs trouvent l'existence d'une relation de long terme entre la croissance économique et les dépenses totales de santé et les dépenses de santé publique, mais cette relation n'est pas valide entre les dépenses de santé privées et la croissance économique. Selon les résultats de l'analyse de causalité du panel de Dumitrescu Hurlin, ils concluent qu'il n'y a pas de relation de causalité entre les dépenses de santé et la croissance économique. Cependant, il est également déterminé que la croissance économique est la principale cause des dépenses de santé totales, publiques et privées.

A l'aide de la technique des moindres carrés ordinaires modifiés (FMLOS), Omitagun et al. (2016) montrent qu'il existe une relation positive et significative entre les effets interactifs des composantes du capital humain (dépenses d'éducation, dépenses de santé) et la croissance au Nigeria. Dans le même pays, des auteurs ont trouvés que les dépenses d'éducation et de santé influencent positivement sur la croissance (Ojo et Oshikoya, 1995 ; Adamu, 2003 ; Mba et al, 2013). Dans le même ordre d'idée, Poças (2014) conclut que l'éducation et la santé contribuent à la croissance économique. En subdivisant le capital humain éducatif en enseignement supérieur, enseignement secondaire et enseignement primaire, (Wang et Liu, 2016) montrent que l'enseignement supérieur a un effet positif sur la croissance économique, cependant l'enseignement primaire et secondaire n'ont pas d'impact significatif. En plus, l'espérance de vie est corrélée positivement à la croissance du PIB. Gyimah-Brempong et al (2006) découvrent dans leur étude que l'éducation a un effet positif et significatif sur la croissance dans les pays africains et que l'élasticité de la croissance du capital humain est deux fois plus élevée que l'impact sur la croissance de l'investissement en capital humain. Par contre d'autres auteurs ont trouvé des résultats contraires. Lawanson, 2009 et Jaiyeoba, 2015, avancent que le budget alloué à la santé à la scolarisation dans l'enseignement primaire a une influence négative sur la croissance. Dans un modèle VAR, Keho (2007) conclut que les dépenses publiques totales et d'éducation n'ont pas d'impact significatif sur le Produit Intérieur Brut (PIB), que l'évolution du PIB influence positivement les dépenses publiques dans le secteur de la santé.

Au sortir de cette revue de littérature théorique comme empirique, nous retenons que les auteurs mesurent différemment le capital humain afin de mesurer son effet sur la croissance économique. Aussi, plusieurs méthode d'estimation sont utilisées. Notre contribution à la recherche scientifique est tout d'abord de prendre en compte le cas spécifique du Mali qui a mis en place plusieurs programme d'investissement dans les secteurs de l'éducation et de la santé. Nous prenons ces dépenses en éducation et en santé pour voir si elle agit positivement sur la croissance économique dans le court terme et dans le long terme.

\section{Méthodologie et source de données}

Nous présentons la specification de notre modèle avant de préciser la source de nos données.

\subsection{Spécification du modèle}

Notre modèle économétrique cherche à étudier l'effet des dépenses publiques en capital humain sur la croissance économique au Mali. Nous partons du modèle de croissance de Solow des travaux de Barro et d'Aschauer, qui intègrent l'investissement public dans la fonction de production. Ce modèle de base se présente comme suit : 
$Y=F(K, L, G)$

Avec Y le produit intérieur Brut (PIB), K est l'investissement privé, L la main d'œuvre et G les investissements publics. Nous nous intéressons aux dépenses publiques en capital humain qui sera capté par les dépenses publiques d'éducation et de santé auxquelles nous ajouterons des variables de contrôle. Le modèle ainsi spécifié sous la forme linéaire est le suivant :

$\ln P I B=\delta_{0}+\delta_{1} \ln D E P E D U+\delta_{2} \ln D E P S A N T+\delta_{3} \ln P O A C T+\delta_{4} \ln E V I E+\delta_{5} \ln M O R T+$ $\delta_{6} \operatorname{lnOUVC}+\mu$

Avec ln le logarithme népérien, DEPEDU les dépenses publiques d'éducation, DEPSANT les dépenses publiques de santé, POPACT la population active, EVIE l'espérance de vie à la naissance, MORT le taux de mortalité, OUVC l'ouverture commerciale, $\delta_{0}$ la constance du modèle et $\mu$ le terme d'erreur. Les paramètres $\delta_{1}$ à $\delta_{6}$ mesurent les élasticités du PIB par rapport aux différentes variables.

\subsection{Sources des données}

Les données utilisées pour les estimations économétriques proviennent de la base des données de la Banque Mondiale Ces données sont des séries chronologiques annuelles qui couvrent la période de 1986 -2018. Cela veut dire que notre échantillon comporte 33 observations.

\section{Estimation du modèle et présentation des résultats}

La première étape dans la modélisation des séries chronologiques consiste à étudier leur stationnarité. Les résultats des tests de stationnarité permettent de définir le modèle adéquat pour estimer l'effet des variables explicatives sur la variable à expliquer.

\subsection{Résultats des tests de stationnarité}

Nous avons utilisé le test de stationnarité de Dicky Fuller Augmenté (ADF) qui est l'un des test le plus utilisé par les travaux de recherche dans l'étude de la stationnarité des séries chronologiques. Les résultats sont dans le tableau ci-dessous:

Tableau $\mathrm{N}^{\circ}$ 1: Test ADF de stationnarité des variables

\begin{tabular}{llll}
\hline Variables & $\begin{array}{l}\text { Stationnarité à } \\
\text { niveau }\end{array}$ & $\begin{array}{l}\text { Stationnarité } \\
\text { différence première }\end{array}$ & $\begin{array}{l}\text { en } \\
\text { Ordre } \\
\text { d'intégration }\end{array}$ \\
\hline $\begin{array}{l}\text { Dépenses d'éducation } \\
\text { Dépenses de santé }\end{array}$ & $-3,264047^{*}$ & $-5,765129^{* * * *}$ & $\mathrm{I}(1)$ \\
$\begin{array}{l}\text { Espérance de vie } \\
\text { Ouverture commerciale }\end{array}$ & $-5,440596^{* * *}$ & & $\mathrm{I}(0)$ \\
PIB & & $-9,716045^{* * *}$ & $\mathrm{I}(0)$ \\
Population active & & $-6,273441^{* * * *}$ & $\mathrm{I}(1)$ \\
Taux de mortalité & $-3,875377^{* *}$ & & $\mathrm{I}(1)$ \\
& $-5,013480^{* * *}$ & & $\mathrm{I}(0)$ \\
\hline
\end{tabular}

Source : Auteurs à partir des estimations sous EVIEWS 10

L'application des tests $\mathrm{ADF}$ de racine unitaire sur les séries étudiées montrent que certaines variables ne sont pas stationnaires à niveau. Ce qui conduit à rejeter l'hypothèse de 
stationnarité pour toutes les séries à niveau. Puisque les variables sont non stationnaires à niveau, on passe à des tests sur les variables transformées en différence première. Les résultats rapportés montrent qu'après avoir différencié les variables une fois, toutes les variables ont été confirmées comme étant stationnaires. Le test Dickey et Fuller Augmentés appliqués à la première différence de la série de données rejettent l'hypothèse du non stationnarité pour toutes les variables utilisées dans cette étude. Il est donc utile de conclure que toutes les variables sont intégrées d'ordre un (1). Donc aucune série n'est intégrée d'ordre deux I(2) ou plus, ce qui est primordial pour l'application de l'ARDL. En effet, le modèle ARDL, présenté par Pesaran et Shin (1999) et développé par Pesaran et al (2001), est une technique qui englobe certains avantages. Premièrement, il permet d'étudier, à la fois, l'effet de court terme et de long terme. Deuxièmement, il permet de donner de meilleures estimations dans des échantillons de petite taille, comme le cas pour notre échantillon (33 observations). L'équation de base est de la forme suivante :

$\Delta y_{t}=\alpha_{0}+\sum_{i=1}^{p} \alpha_{1 i} \Delta y_{t-1}+\sum_{i=0}^{q} \alpha_{2 i} \Delta x_{t-1}+\beta_{1} y_{t-1}+\beta_{2} x_{t-1}+\varepsilon_{i}$

Avec : $y$ la variable à expliquer ; $x$ t le vecteur des variables explicatives $\alpha_{1 i}$ et $\alpha_{2 i}$ les effets de court terme $; \beta_{1}$ et $\beta_{2}$ les effets de long terme $\Delta$ la différence première $; \alpha_{0}$ la constante $; \varepsilon_{i}$ le terme d'erreur.

En appliquant la forme générale du modèle ARDL sur les variables de notre modèle spécifié, nous obtenons :

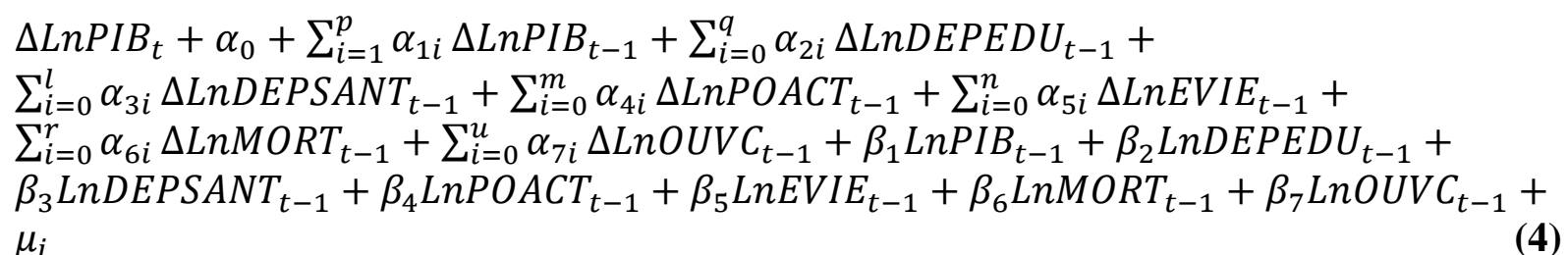

Avec $\alpha_{1 i}$ à $\alpha_{7 i}$ les coefficient qui captent les effets de court terme ; $\beta_{1}$ à $\beta_{7}$ les coefficients qui captent les effets de long terme $\Delta$ la différence première ; $\alpha_{0}$ la constante $; \mu_{i}$ le terme d'erreur et $\mathrm{t}=$ année

\subsection{Estimation du modèle ARDL}

Dans les modèles autoregressifs, plusieurs modèle ARDL peuvent être estimés selon le nombre de retard définie. Il faut ainsi choisir un modèle optimal reposant sur le principe de la parcimenie. Ce principe se fonde sur le choix du modèle qui fournit les critères d'information d'Ackaik et de Schwars sont les plus minimaux. Le logiciel Eviews 10 utilisé dans cette recherche nous a permis de retenir le modèle $\operatorname{ARDL}(1,2,2,0,2,2,1)$ comme le modèle optimal parmi les 20 meilleurs modèles

En outre, le coefficient de détermination R2 qui est de 0,99 nous confirme le bon ajustement du modèle. Cela veut dire que les variables explicatives, prises dans notre modèle économétrique, contribuent à l'explication de la variable dépendante (PIB) à l'ordre de 99\% (voir figure 1) et tableau $\mathrm{N}^{\circ} 2$ ci-dessous). 
Figure 1 : valeurs du critère d'information Akaike pour les 20 meilleurs modèles

Akaike Information Criteria (top 20 models)

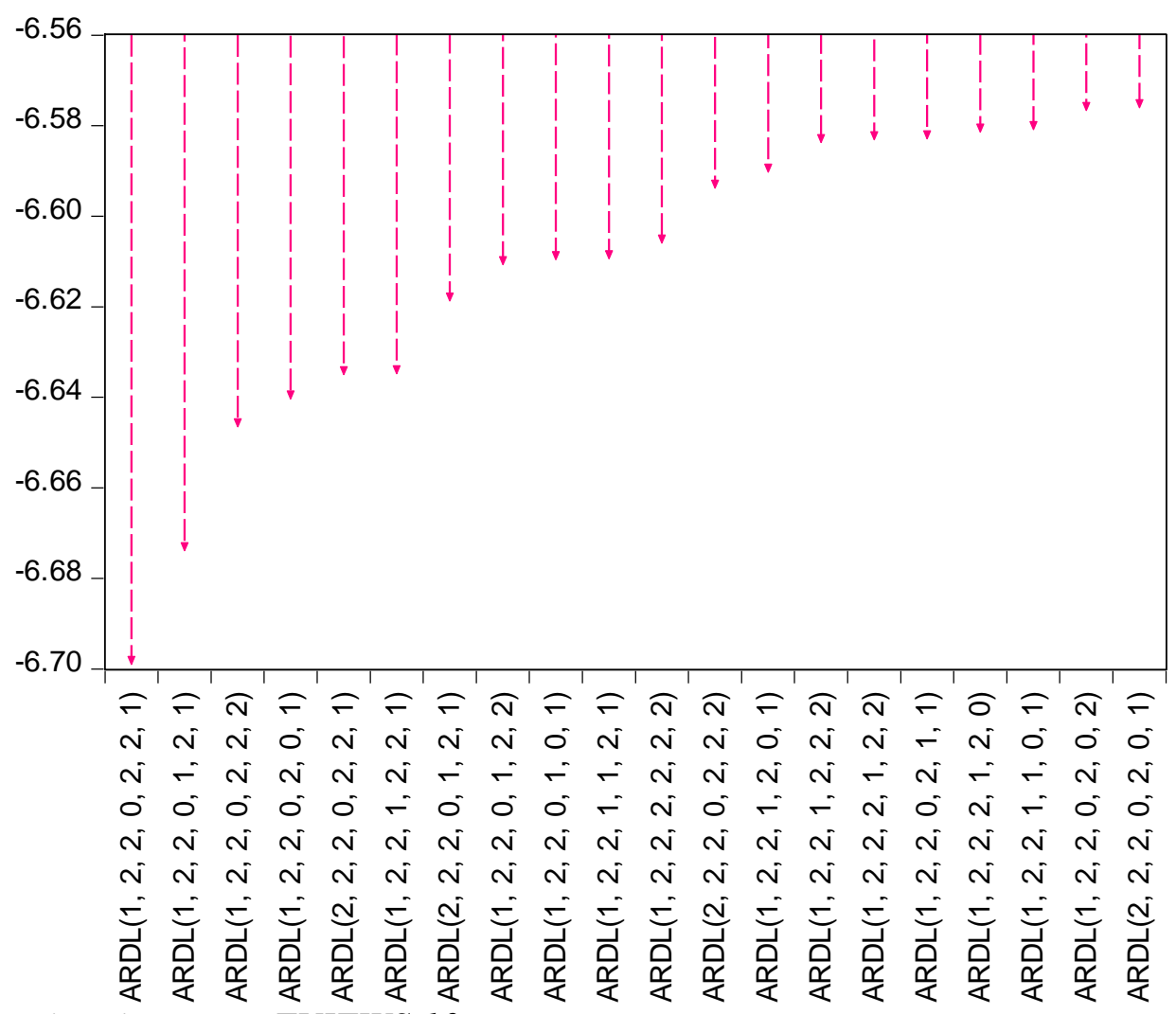

Auteurs : estimations sous EVIEWS 10

Tableau $\mathbf{N}^{\circ} 2$ : Estimation du modèle ARDL $(1,2,2,0,2,2,1)$

\begin{tabular}{lllll}
\hline \hline Variable & Coefficient & Std. Error & t-Statistic & Prob.* \\
\hline \hline LnPIB(-1) & $-0,157920$ & 0,220001 & $-0,717815$ & 0,4847 \\
LnDEPSANT & 0,102059 & 0,024908 & 4,097436 & 0,0011 \\
LnDEPSANT(-1) & 0,022597 & 0,037039 & 0,610078 & 0,5516 \\
LnDEPSANT(-2) & 0,120320 & 0,035911 & 3,350530 & 0,0048 \\
LnDEPEDU & 0,025107 & 0,010565 & 2,376512 & 0,0323 \\
LnDEPEDU(-1) & 0,024421 & 0,009131 & 2,674658 & 0,0181 \\
LnDEPEDU(-2) & 0,039153 & 0,010445 & 3,748425 & 0,0022 \\
LnEVIE & $-16,67144$ & 2,655368 & $-6,278390$ & 0,0000 \\
LnPOACT & $-0,151967$ & 0,202533 & $-0,750334$ & 0,4655 \\
LnPOACT (-1) & 0,508567 & 0,089100 & 5,707800 & 0,0001 \\
LnPOACT (-2) & 0,162216 & 0,141544 & 1,146047 & 0,2710 \\
LnOUVC & 0,073768 & 0,040140 & 1,837757 & 0,0874 \\
LnOUVC(-1) & $-0,017436$ & 0,047250 & $-0,369024$ & 0,7176 \\
LnOUVC(-2) & $-0,080292$ & 0,048230 & $-1,664783$ & 0,1182 \\
LnMORT & $-18,66340$ & 2,785773 & $-6,699540$ & 0,0000 \\
LnMORT(-1) & 12,53910 & 1,947680 & 6,437967 & 0,0000 \\
C & 41,31360 & 6,266588 & 6,592678 & 0,0000 \\
\hline \hline
\end{tabular}




$\begin{array}{llll}\text { R-squared } & 0,999257 & \text { Mean dependent var } & 10,29389 \\ \text { Adjusted R-squared } & 0,998408 & \text { S,D, dependent var } & 0,183133 \\ \text { S,E, of regression } & 0,007307 & \text { Akaike info criterion } & -6,698021 \\ \text { Sum squared resid } & 0,000748 & \text { Schwarz criterion } & -5,911641 \\ \text { Log likelihood } & 120,8193 & \text { Hannan-Quinn criter, } & -6,441681 \\ \text { F-statistic } & 1176,763 & \text { Durbin-Watson stat } & 2,567413 \\ \text { Prob(F-statistic) } & 0,000000 & & \end{array}$

Source : Auteurs à partir des estimations sous EVIEWS 10

\subsection{Test de cointégration (Bound Test)}

Cette étape a pour objectif de vérifier l'existence d'une relation de long terme entre les variables de notre modèle spécifié. A ce sujet, nous appliquons le test aux bondes de Pesaran qui permet de tester la cointégration entre des variables d'ordre d'intégration différents (I (0) ou I (1)). Le principe de ce test repose sur la comparaison de la valeur de Ficher statistique avec la valeur de la bonde inférieur (I0) et de la bonde supérieur (I1) pour les différents seuils de significativité. La cointégration existe lorsque la valeur de la statistique de Ficher est supérieur à la valeur de la bonde supérieur. Les résultats de la procédure « Bounds test » montrent que la statistique de Fisher $(\mathrm{F}=9,805302)$ est supérieure à la borne supérieure de l'intervalle des valeurs critiques correspond au niveau de d'erreur de $1 \%$. Donc on rejette l'hypothèse d'absence de relation de long terme, nous concluons qu'il existe une relation de cointégration entre les variables de notre modèle. Nous pouvons donc estimer la relation de court terme et de long terme.

Tableau $\mathbf{N}^{\circ} 3$ : test au bond de cointégration

\begin{tabular}{lllll}
\hline F-Bounds Test & & \multicolumn{3}{c}{ Null Hypothesis : No levels relationship } \\
\hline Test Statistic & Value & Signif. & I(0) & I(1) \\
\hline F-statistic & 9,805302 & $10 \%$ & 1,99 & 2,94 \\
k & 6 & $5 \%$ & 2,27 & 3,28 \\
& & $2,5 \%$ & 2,55 & 3,61 \\
& & $1 \%$ & 2,88 & 3,99 \\
\hline
\end{tabular}

Source : Auteurs à partir des estimations sous EVIEWS 10

\subsection{Estimation de la relation de court terme ARDL (1, 2, 2, 0, 2, 2, 1)}

Les résultats de l'estimation de la relation de court terme montrent que le coefficient de correction d'erreur est négatif $(-1,15)$ et significatif au seuil de $1 \%(\mathrm{P}=0,00)$, ce qui est très confortable pour interpréter la relation de court terme entre les variables. En effet, ce coefficient à correction d'erreur nous indique que l'ajustement vers un équilibre de long terme se corrige à $115 \%$ par année. En d'autre termes, les résultats du tableau ci-dessous montrent que l'effet des dépenses en capital humain sur la croissance économique (PIB) est assez important même si le signe de cet effet change d'une année à une autre. Une augmentation des dépenses en santé de $1 \%$ entraine une augmentation de $0,10 \%$ le PIB et une réduction de ce dernier l'année suivante de $0,12 \%$. C'est le même constat pour les dépenses d'éducation qui, augmentées de $1 \%$ augmenterais le PIB de $0,02 \%$ la même année et le réduirai le PIB de l'année suivante de $0,03 \%$. Cette situation montre simplement que les dépenses en capital humain sont favorables à la croissance économique à court terme. Ces résultats vont dans le même sens que la théorie du capital humain et la littérature empirique (Samoud et Idriss, 2021 ; Piabuo et Tieguhong, 
2017). Néanmoins le sens des relations change de signe d'une année à une autre. En effet, les dépenses en capital humain d'aujourd'hui ont un effet plus important sur la croissance économique que les années à venir. Cela s'explique dans le cas malien du fait que les investissements en capital humain sont beaucoup plus orientés dans le fonctionnement des structures que les dépenses d'investissements qui ont plus d'effet de long terme. Les variables telles la population active et le taux de mortalité ont un effet négatif sur la croissance économique au Mali. L'ouverture commerciale à un effet positif à court terme sur la croissance économique.

Tableau $N^{\circ} 4$ : Résultat de l'estimation de court terme

\begin{tabular}{lllll}
\hline \hline Variable & Coefficient & Std. Error & t-Statistic & Prob. \\
\hline \hline D(Dépenses de santé) & $0,102059^{* * *} *$ & 0,012933 & 7,891135 & 0,0000 \\
D(Dépenses de santé (-1)) & $-0,120320^{* * *}$ & 0,016714 & $-7,198521$ & 0,0000 \\
D(Dépenses d'éducation) & $0,025107^{* * *}$ & 0,004733 & 5,304866 & 0,0001 \\
D(Dépenses d'éducation (-1)) & $-0,039153^{* * *}$ & 0,004686 & $-8,355087$ & 0,0000 \\
D(Population active) & $-0,151967 * * *$ & 0,036339 & $-4,181993$ & 0,0009 \\
D(Population active(-1)) & $-0,162216^{* * *}$ & 0,040834 & $-3,972596$ & 0,0014 \\
D(ouverture commerciale) & $0,073768^{* * *}$ & 0,019358 & 3,810698 & 0,0019 \\
D(Ouverture commerciale(-1)) $0,080292^{* * *}$ & 0,018461 & 4,349204 & 0,0007 \\
D(taux brut de mortalité) & $-18,66340^{* * *}$ & 1,595200 & $-11,69972$ & 0,0000 \\
CointEq(-1)* & $-1,157920^{* * *}$ & 0,106747 & $-10,84729$ & 0,0000 \\
\hline \hline R-squared & 0,887928 & Mean dependent var & 0,019103 \\
Adjusted R-squared & 0,839897 & S,D, dependent var & 0,014911 \\
S,E, of regression & 0,005966 & Akaike info criterion & $-7,149634$ \\
Sum squared resid & 0,000748 & Schwarz criterion & $-6,687057$ \\
Log likelihood & 120,8193 & Hannan-Quinn criter, & $-6,998845$ \\
Durbin-Watson stat & 2,567413 & & & \\
\hline \hline
\end{tabular}

*** = significativité au seuil de $1 \%$

Source : Auteurs à partir des estimations sous EVIEWS 10

\subsection{Estimation de la relation de long terme $(1,2,2,0,2,2,1)$}

L'estimation de la relation de long terme, présentée dans le tableau suivant, montre que les coefficients des dépenses en capital humain sont positifs sur la croissance économique et tous significatifs au seuil de $1 \%$. Une augmentation des dépenses publiques respectivement de santé et d'éducation augmente la croissance économique respectivement de $0,2 \%$ et $0,07 \%$. Ces résultats sont conformes à ceux trouvés par El Houda Sadi et Rezine (2021) sur le cas de l'Egypte et de Samoud et Driss (2021) sur le cas du Maroc. Au niveau des études faites sur des groupes de pays, nos résultats sont aussi conformes à ceux trouvés par Piabuo et Tieguhong (2017) dans leur analyse des impacts des dépenses de santé en zones CEMAC et cinq autres pays Africains. On remarque dans notre étude que l'effet des dépenses en santé sur la croissance économique est plus élevé que celui des dépenses en éducation. Cela est aussi vérifié par plusieurs auteurs (Omitagun et Al.,2016 ; Piabuo et Tieguhong, 2021). En somme, notre étude montre que les dépenses en capital humain sont positivement corrélées avec la croissance économique au Mali. Le sens de ces relations est conforme à la théorie du capital humain et confirmé par plusieurs. Au niveau des variables de contrôle, la population active a un effet 
positif et significatif sur la croissance économique de long terme. L'espérance de vie à la naissance et le taux de mortalité ont un effet négatif sur la croissance économique.

Tableau $\mathrm{N}^{\circ} 5$ : Résultat de l'estimation de long terme

\begin{tabular}{|c|c|c|c|c|}
\hline Variable & Coefficient & Std. Error & t-Statistic & Prob. \\
\hline Dépenses de santé & $0,211565^{* * *}$ & 0,063070 & 3,354444 & 0,0047 \\
\hline Dépenses d'éducation & $0,076587 * *$ & 0,023864 & 3,209328 & 0,0063 \\
\hline Espérance de vie & $-14,39775^{* * *}$ & 2,812611 & $-5,118997$ & 0,0002 \\
\hline Population active & $0,448058 * * *$ & 0,130023 & 3,445991 & 0,0039 \\
\hline Ouverture commerciale & $-0,020693$ & 0,074758 & $-0,276797$ & 0,7860 \\
\hline Taux de mortalité & $-5,289053 * * *$ & 0,741808 & $-7,129952$ & 0,0000 \\
\hline $\mathrm{C}$ & $35,67915 * * *$ & 4,426523 & 8,060311 & 0,0000 \\
\hline
\end{tabular}

Source : Auteurs à partir des estimations sous EVIEWS 10

\subsection{Tests de robustesse du modèle}

La validité de notre modèle estimé et celles des résultats obtenus des relations de court terme et de long terme nécessite une confirmation à l'aide d'un ensemble d'hypothèse. Nous vérifions les hypothèses sur la corrélation des erreurs, l'hétéroscédasticité, la normalité, la spécification et la stabilité des coefficients. Dans le tableau ci-dessous, la probabilité de la statistique pour les 4 tests est supérieure à $5 \%$. Ce qui signifie que l'hypothèse H0 est acceptée dans tous ces tests. Les erreurs ne sont donc pas corrélées, ne sont pas homoscédastique et leur distribution suit une loi normale. Notre modèle est par conséquent bien spécifié. Aussi, la stabilité des coefficients de notre modèle ARDL $(1,2,2,0,2,2,1)$ dans le temps est validé par les tests de CUSUM et CUSUMQ, car la courbe ne sort pas du corridor dans ces deux tests (figure cidessous). Nous pouvons donc confirmer la robustesse de notre modèle ARDL (1, 2, 2, 0, 2, 2, 1) estimé.

Tableau $\mathbf{N}^{\circ}$ 6: test d'hypothèse du modèle ARDL (1, 2, 2, 0, 2, 2, 1)

\begin{tabular}{|c|c|c|c|}
\hline Hypothèse vérifiée & Test appliqué & Statistique & Probabilité \\
\hline $\begin{array}{l}\text { Autocorrélation } \\
\text { erreurs }\end{array}$ & Breusch-Godfrey & 1,952674 & 0,1844 \\
\hline Hétéroscédasticité & Arch & 2,551913 & 0,1214 \\
\hline Normalité & Jarque-Bera & 3,16081 & 0,5701 \\
\hline Spécification & Ramsey & 0,001779 & 0,9670 \\
\hline
\end{tabular}

Source : Auteurs à partir des estimations sous EVIEWS 10 


\section{Fig 2 : Test de CUSUM et de CUSUMQ}
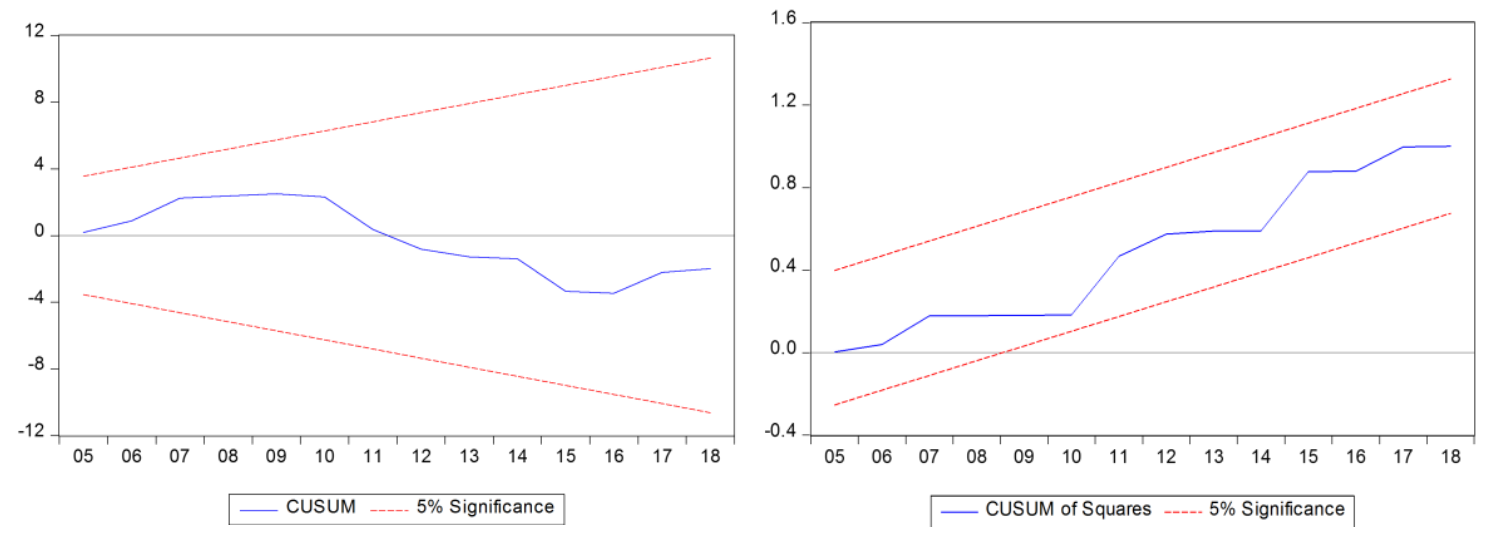

Source : Auteurs à partir des estimations sous EVIEWS 10

\section{Conclusion et implication de politiques économiques}

Selon les théoriciens du capital humain, notamment ceux de la croissance endogène, la croissance économique est fortement liée à l'accumulation du capital humain. Les dépenses publiques en soi sont également en faveur de la croissance économique. Ce papier avait pour objectif de mesurer l'effet des dépenses publiques en capital humain sur la croissance économique. Ces dépenses en capital humain représentent aussi l'aspect qualité du capital humain. Les estimations ARDL de court terme et de long terme montrent que les dépenses en capital humain augmentent la croissance économique au Mali. En effet, pour le court terme les dépenses en capital humain sont beaucoup favorable à la croissance économique de l'année en cours et moins favorable pour les années qui suivent. Cela s'explique dans le cas malien par le fait que peu de dépenses publiques alloué aux investissements de long terme. Dans le long terme, les dépenses en capital humain ont un effet positif sur la croissance économique au Mali ; cet effet est plus important pour les dépenses de santé que les dépenses d'éducation. Il est à noter que l'espérance de vie à la naissance et le taux de moralité réduisent la croissance économique malienne. La population active quant à elle augmente fortement la croissance économique.

Au regard de ces résultats, les politiques économiques doivent permettre d'orienter les dépenses en capital humain vers les investissements de long terme. En effet, les dépenses en capital humain au Mali, portent plus sur les dépenses de fonctionnement que celles d'investissements, c'est ce qui explique leur effet de moins en moins important quand l'on tend vers le long terme. Si les dépenses s'orientent plus dans les investissements en capital humain, cela améliorent la qualité du capital humain (éducation et santé) d'une part, et d'autre part, valorise la population active à travers la formation et l'emploi.

\section{Bibliographie}

[1] P. A. Adamu, 'The impact of human capital formation on economic development in Nigeria: An error correction approach"' The Nigerian economic society, 2003, vol. 2, p. 53-77.

[2] O.A. Adelowokan, "Growth effect of education and health expenditure in Nigeria (1970-2010)'. African Journal of Scientific Research, 2012, vol. 10, no 1, p. 510-528.. 
[3] R.J. Barro, 'Economic growth in a cross section of countries', The quarterly journal of economics, 1991, vol. 106, no 2, p. 407-443.

[4] R.J. Barro, 'Determinants of economic growth: a cross-country empirical study'. Lionel Robbins Lectures. In : Cambridge, MA: MIT Press. 1997.

[5] R.J. Barro, 'Determinants of economic growth: implications of the global evidence for Chile'. Cuadernos de economía, 1999, p. 443-478.

[6] B. Bathily, et A.B. Gueye, 'Dépenses publiques de santé et croissance économique en Afrique Subsaharienne: une analyse de long terme par la méthode des Panels var (Public Health Expenditure and Economic Growth in Sub-Saharan Africa: A Long-Term Analysis Using the Var Panel Method)'". Available at SSRN 3772827, 2021.

[7] G.S. Becker, «Human capital: a theoretical and empirical analysis, with special reference to education », National Bureau of Economic Research publications, 1964, New York.

[8] J. R. Behrman, et J. C. Knowles, 'Population and reproductive health: An economic framework for policy evaluation'. Population and development review, 1998, p.697-737.

[9] J. Benhabib et M. M. Spiegel, ' The role of human capital in economic development evidence from aggregate cross-country data', Journal of Monetary economics, 1994 Vol. 34(2), p.143-173.

[10] D. Bloom et D. Canning, 'The health and poverty of nations : from theory to practice'". Journal of human development, 2003, vol. 4, no 1, p. 47-71.

[11] E. F. Denison, 'United States economic growth', The Journal of Business, 1962, vol. 35, no 2, p. 109-121.

[12] A. Diagne, 'Investir sur le capital humain : éducation et santé'. African development review, 2007, vol. 19, no 1, p. 163-199.

[13] H. Dinçer et S. Yuksel, 'Serhat. Identifying the causality relationship between health expenditure and economic growth: An application on E7 countries', Journal of Health Systems and Policies, 2019, vol. 1, no 1, p. 5-23.

[14] N. El Houda Sadi et O. Rezine, "Capital humain et croissance economique: Une analyse empirique de donnees de panel sur la periode 1975-2015', les cahiers du cread, 2021, vol. 37, no 1, p. 149-172.

[15] I. Fatiha, D. Brahim et K. Mourad, 'Education, Santé et croissance économique en Algérie : Étude économétrique via le modèle ARDL", 2021, vol. 12, no 1, p. 753-769.

[16] M. Grossman, 'The human capital model of the demand for health'. NBER Working paper, 1999, no w7078.

[17] K. Gyimah-Brempong, O. Paddison et W. Mitiku, 'Higher education and economic growth in Africa'. The Journal of Development Studies, 2006, vol. 42, no 3, p. 509-529.

[18] R. E. Hall, et C.I. Jones, 'Why do some countries produce so much more output per worker than others?'” The quarterly journal of economics, 1999, vol. 114, no 1, p. 83-116.

[19] A. O. Hirschman, et G. Sirkin, 'Investment criteria and capital intensity once again'. The Quarterly Journal of Economics, 1958, vol. 72, no 3, p. 469-471.

[20] P. Howitt et P. Aghion, "Capital accumulation and innovation as complementary factors in long-run growth'. Journal of Economic Growth, 1998, vol. 3, no 2, p. 111-130.

[21] S. V. Jaiyeoba, 'Human capital investment and economic growth in Nigeria'. African Research Review, 2015, vol. 9, no 1, p. 30-46.

[22] Y. Keho, ''La dimension sociale du developpement durable en Cote d'Ivoire: le role de la croissance et des depenses sociales'. Cellule d'Analyse de Politiques Economiques du CIRES, 2009.

[23] O. I. Lawanson, 'Human capital investment and economic development in Nigeria: The role of health and education". 2009.

[24] R. E. Lucas JR, ' On the mechanics of economic development'. Journal of monetary economics, 1988 , vol. 22 , no 1, p. 3-42.

[25] A. Maddison, "Growth and slowdown in advanced capitalist economies: techniques of quantitative assessment'. Journal of economic literature, 1987, vol. 25, no 2, p. 649-698.

[26] N. G. Mankiw, D. Romer, et D. N. Weil, 'A contribution to the empirics of economic growth'. The quarterly journal of economics, 1992, vol. 107, no 2, p. 407-437.

[27] I. C. Mba, E. I. Mba, J. E. Ogbuabor, et al. 'Human capital development and economic growth in Nigeria'. Journal of Economics and Sustainable Development, 2013, vol. 4, no 18, p. 48-52.

[28] J. Mincer, 'Schooling, Experience, and Earnings'. Human Behavior \& Social Institutions No. 2. 1974.

[29] S. Mohapatra, 'Economic growth, public expenditure on health and IMR in India: An econometric investigation of causal linkages'. International Journal of Social Economics, 2017.

[30] O. Ojo, et T. Oshikoya, 'Determinants of long-term growth: Some African results'. Journal of African economies, 1995, vol. 4, no 2, p. 163-191. 
[31] O. Olonade, T. I. Olawande, O.J. Alabi, et al. 'Maternal mortality and maternal health care in Nigeria: Implications for socio-economic development'. Open access Macedonian journal of medical sciences, 2019, vol. 7, no 5, p. 849.

[32] O. Omitogun, A. M. Osoba, et S. A. Tella, ' An Interactive Effect of Human Capital Variables and Economic Growth in Nigeria'. Acta Universitatis Danubius. Economica, 2016, vol. 12, no 5.

[33] S. M. Piabuo et J. C. Tieguhong, 'Health expenditure and economic growth-a review of the literature and an analysis between the economic community for central African states (CEMAC) and selected African countries'. Health economics review, 2017, vol. 7, no 1, p. 1-13.

[34] A. I. Poças, 'Human capital dimensions-education and health-and economic growth'. 2014.

[35] S. Rebelo, 'Long-run policy analysis and long-run growth'. Journal of political Economy, 1991, vol. 99, no 3, p. 500-521.

[36] P. M. Romer, '’Are nonconvexities important for understanding growth?', 1990.

[37] P. M. Romer, 'Increasing returns and long-run growth'”. Journal of political economy, 1986, vol. 94, no 5, p. $1002-1037$.

[38] P. N. Rosenstein-rodan, 'Problems of industrialisation of eastern and south-eastern Europe'. The economic journal, 1943, vol. 53, no 210/211, p. 202-211.

[39] X. Sala-I-Martin, 'Transfers, social safety nets, and economic growth'. Staff Papers, 1997, vol. 44, no 1, p. 81-102.

[40] A. B. Samoud, et A. S. S. I. Driss, 'Tribution du capital humain à la croissance économique au Maroc : Une analyse économétrique à travers le modèle autorégressif à retards'. Alternatives Managériales Economiques, 2021, vol. 3, no 1, p. 463-480.

[41] M. Schiff et Y. Wang, 'Education, governance and trade-related technology diffusion in Latin America'. Available at SSRN 515922, 2004.

[42] T. W. Schultz, 'Investment in human capital'. The American economic review, 1961, vol. 51, no 1, p. 117.

[43] A. Smith, "An inquiry into the nature and causes of the wealth of nations" : Volume One. London : printed for W. 1776, Strahan; and T. Cadell, 1776.

[44] R. M. Solow, 'A contribution to the theory of economic growth'. The quarterly journal of economics, 1956, vol. 70, no 1, p. 65-94. 